EXPERIÈNCIES 



\title{
La recepción de los productos accesibles y la formación de subtituladores: Personas sordas en el aula de SPS
}

\author{
Laura Mejías Climent \\ Universitat Jaume I. Departament de Traducció i Comunicació \\ Campus del Riu Sec, E \\ 12071 Castelló de la Plana \\ 1mejias@uji.es \\ ORCID: 0000-0003-2933-7195
}

\section{Resumen}

Este artículo detalla un proyecto de innovación educativa desarrollado en la Universitat Jaume I para la asignatura de accesibilidad del grado en Traducción e Interpretación. Dada la dificultad de concienciar al alumnado de las características tan heterogéneas y particulares que presenta el colectivo de personas sordas, que serán los receptores potenciales de la futura producción accesible, el objetivo principal fue poner en contacto a los estudiantes con personas sordas que pudieran transmitirles de primera mano sus expectativas y experiencias como consumidores audiovisuales. El proyecto abarcó la valoración de algunos subtítulos elaborados según la norma UNE 153010 (2012) y entrevistas semiestructuradas con las personas visitantes. También se realizaron actividades previas y posteriores a la sesión en el aula con las que se completó el cumplimiento de los objetivos.

Palabras clave: accesibilidad; subtitulación para personas sordas (SPS); competencia interpersonal; aprendizaje-servicio

Abstract. Deaf and hard of hearing users participation in the accessibility classroom: a complementary experience to subtitling training

This paper presents an innovative teaching project developed at Universitat Jaume I as part of an accessibility course included in the Translation and Interpreting degree. The deaf and hard of hearing community is characterized by their heterogeneity. However, students are not always aware of the wide range of profiles and needs that they will encounter. Thus, the aim of this project was to bring together students - as future professionals - and deaf people - as the future audience of the subtitling production-, to let the latter share their real expectations and experiences as audiovisual consumers. The project included the assessment of some subtitles following the Spanish standard UNE 153010 (AENOR 2012) as well as semi-structured interviews with the visiting deaf people. Some previous and subsequent activities complemented the contents of this session to ensure that all the goals were attained.

Keywords: accessibility; subtitling for the D/deaf and hard of hearing (SDH); interpersonal competence; service-learning 


\author{
Sumario \\ 1. Introducción 4. Resultados \\ 2. La SPS: competencias y alcance \\ 5. Conclusiones \\ 3. Metodología del proyecto de \\ Referencias bibliográficas \\ innovación docente
}

\title{
1. Introducción
}

La accesibilidad es un ámbito de reciente incorporación a la docencia universitaria, estrechamente relacionado con la Traducción Audiovisual (TAV) y cuya demanda en el mercado es cada vez mayor, dadas las normativas y regulaciones, tanto estatales como europeas, ${ }^{1}$ que promueven la emisión de cada vez más contenido accesible en los medios, para garantizar los derechos de información y entretenimiento de todos los ciudadanos. En este artículo, nos centraremos en una de las modalidades de TAV accesibles: la subtitulación para personas sordas y con deficiencias auditivas (SPS), para desarrollar un proyecto de innovación docente en el que converjan tanto el alumnado en formación como los espectadores potenciales del producto subtitulado.

A partir de la incorporación de la subtitulación al mercado televisivo en la década de los setenta (Ivarsson y Carroll 1998), ha sido evidente la rápida evolución del medio y, por tanto, de las características de esta modalidad de TAV. Nos remitimos a Díaz Cintas (2010), Tamayo Masero (2015) y Reverter Oliver (2019) para una revisión del desarrollo de esta modalidad de TAV accesible. Del mismo modo, las propuestas didácticas para su docencia en el ámbito de estudios superiores han evolucionado en sus aún escasos años de recorrido, desde que se reconociera la necesidad de formación en accesibilidad en general, en la Ley 51/2003, de 2 de diciembre, de Igualdad de oportunidades, no discriminación y accesibilidad universal de las personas con discapacidad (LIONDAU), así como en el I Plan Nacional de Accesibilidad «Diseño para todos» 2004-2012, del Ministerio de Trabajo y Asuntos Sociales (2003) (Badia Cardús y Matamala 2007).

Desde la puesta en marcha de la formación en accesibilidad y, entre muchas otras, las prácticas descritas por Neves (2005) o la propuesta formulada por los citados Badia Cardús y Matamala en 2007 para la formación en este ámbito (audiodescripción, SPS y lengua de signos), han sido varios los planteamientos y enfoques para la mejora de la docencia de estas modalidades de TAV, como es el caso de De Higes Andino y Cerezo Merchán (2018), con una propuesta de criterios y rúbricas de evaluación; Cerezo Merchán y De Higes Andino (2013), empleando el trabajo colaborativo; o Tamayo Masero (2016), que desarrolló un

1. Ley 7/2010, de 31 de marzo, General de la Comunicación Audiovisual; Real Decreto Legislativo 1/2013, de 29 de noviembre, por el que se aprueba el Texto Refundido de la Ley General de derechos de las personas con discapacidad y de su inclusión social; Convención de las Naciones Unidas sobre el derecho de las personas con discapacidad. 
proyecto basado en la metodología de aprendizaje-servicio en la SPS a partir del cual hemos diseñado la experiencia descrita en las siguientes páginas.

Uno de los aspectos que han evolucionado en la didáctica de la Traducción desde sus orígenes, a mediados del siglo pasado, ha sido la adopción de modelos didácticos cognitivo-constructivistas centrados en el estudiante como actor principal de su aprendizaje, así como la apuesta por un enfoque docente en estrecha relación con el mundo profesional (Cerezo Merchán y De Higes Andino 2013: 66). Además de un enfoque estrechamente relacionado con el mundo laboral, aspecto de vital importancia para la inserción profesional, consideramos también de gran importancia la concienciación del alumnado sobre el objetivo real del producto que aprenden a elaborar en las aulas. «Only by knowing the distinctive features of the target audience will translators be reasonably aware of the possible effects their work may produce on their receivers» (Neves 2005: 123). En el caso de la accesibilidad, y como ponen de manifiesto Arumí Ribas y RomeroFresco (2008: 116), una de las competencias más relevantes que han de adquirir los futuros profesionales de la accesibilidad, en relación con el texto y el contexto meta, es la concienciación y el conocimiento sobre las necesidades de los futuros receptores, es decir, entender cuáles son sus dificultades y capacidades al consumir productos audiovisuales.

Esta ha sido la habilidad en la que pretende centrarse en nuestro proyecto. $\mathrm{Si}$ repasamos planes de estudios que contengan asignaturas de accesibilidad (apartado 2), evidentemente, en todos ellos se incluyen las destrezas técnicas, la configuración de los parámetros de espacio y tiempo o la representación de información contextual y efectos sonoros, entre algunas otras cuestiones relativas a los parámetros subtituladores. No tanto es así con los futuros receptores del producto audiovisual subtitulado: aunque en muchos programas docentes se incluya una referencia a la comunidad sorda, en raras ocasiones los alumnos tienen un contacto real, de primera mano, con las personas que habrán de disfrutar de una SPS de calidad. Por ello, este proyecto, llevado a cabo en el marco de los programas de innovación docente de la Universitat Jaume $\mathrm{I}^{2}$ pretende ampliar la formación de los estudiantes de accesibilidad poniéndoles en contacto con personas con problemas de audición que les transmitan sus propias necesidades y expectativas cuando se sientan ante una pantalla.

2. Este proyecto se desarrolló en el seno del Grupo de Innovación Educativa iTRAMA (Innovación Educativa en la Traducción para los Medios Audiovisuales y la Accesibilidad), financiado por la Unitat de Suport Educatiu de la Universitat Jaume I, y en relación con el proyecto de investigación ITACA (Inclusión Social, Traducción Audiovisual y Comunicación Audiovisual), ref. FFI2016-76054-P, 2016-2019, financiado por el Ministerio de Economía y Competitividad de España. Se agradece asimismo la colaboración de la Coordinadora de Proyectos y Servicios de ASPAS-Castellón y de las dos personas con deficiencias auditivas que se prestaron a participar en el proyecto. 


\section{La SPS: competencias y alcance}

La asignatura de accesibilidad TI0952 del grado en Traducción e Interpretación de la Universitat Jaume I abarca dos de las principales modalidades de TAV destinadas a personas con algún tipo de discapacidad sensorial que les impide consumir productos audiovisuales tal como se crearon: la audiodescripción para personas ciegas y la subtitulación para personas sordas (SPS) (Díaz-Cintas, Orero y Remael 2007). La segunda consiste en

presentar en pantalla un texto escrito que ofrece un recuento semántico de lo que se emite en el programa en cuestión, pero no sólo [sic] de lo que se dice, cómo se dice (énfasis, tono de voz, acentos e idiomas extranjeros, ruidos de la voz) y quién lo dice sino también de lo que se oye (música y ruidos ambientales) y de los elementos discursivos que aparecen en la imagen (cartas, leyendas, carteles, etc.). (Pereira Rodríguez 2005)

Como señalábamos, se trata de una práctica relativamente reciente, empleada por primera vez en la televisión pública norteamericana en 1973 y que no llegó a las pantallas españolas hasta principios de los años noventa (Pereira Rodríguez 2005; Díaz-Cintas, Orero y Remael 2007). En este contexto, la formación en SPS comenzó a impartirse cuando el paradigma docente ya se había desplazado desde los modelos de enseñanza transmisionistas al aprendizaje colaborativo y centrado en el protagonismo del estudiantado, cuya formación está estrechamente relacionada con el desarrollo de competencias, promovidas asimismo por la implantación del Espacio Europeo de Educación Superior. Entre dichas competencias, la interpersonal desarrolla un papel muy relevante (Cerezo Merchán y De Higes Andino 2013). Según Tamayo Masero (2016: 330), «en la docencia de esta materia parece necesario hacer hincapié en el desarrollo de la competencia interpersonal y apostar por una metodología que pueda impulsar la adquisición de dicha competencia de forma eficiente».

La interpersonal representa una competencia básica que fomenta, entre otros aspectos, la toma de conciencia, el respeto hacia la diversidad, las capacidades lingüísticas y la participación activa en la comunidad a la que se pertenece (Rodríguez Esteban 2007), aspectos que pueden considerarse básicos para el pleno desarrollo de los profesionales de la SPS y su conocimiento de los espectadores potenciales del producto que aprenden a elaborar. Esta será una de las principales competencias que nos planteamos desarrollar con el presente proyecto de innovación educativa. Asimismo, según el modelo de la competencia traductora de Kelly (2005), la competencia interpersonal abarca la capacidad de interrelacionarse y colaborar con otros profesionales, aspecto que también se incluye en nuestro proyecto mediante el trabajo colaborativo, así como el fomento de la ética y la responsabilidad social, contemplados en el modelo educativo de la Universitat Jaume I (Consejo de Gobierno 2016).

Por otra parte, es innegable la importancia que debe tener en nuestra sociedad la accesibilidad para permitir el acceso generalizado a la información y al entretenimiento (Díaz-Cintas, Orero y Remael 2007), y, de hecho, en los últimos años se 
ha observado una oferta creciente de los productos audiovisuales que incluyen alguna modalidad de TAV accesible (Kelly 2005). Este aumento de la oferta accesible se vio impulsado por la Ley 7/2010, de 31 de marzo, General de la Comunicación Audiovisual, con la cual, «el Estado español transpone la Directiva 2007/65/CE de Servicios de Comunicación Audiovisual del Parlamento Europeo y del Consejo de 11 de diciembre del 2007» (Cerezo Merchán y De Higes Andino 2013: 69). Esta ley estableció un aumento progresivo de las emisiones accesibles, integrando tanto SPS como audiodescripción e interpretación en lengua de signos. Actualmente, la Comisión Nacional de los Mercados y la Competencia emite un informe anual en el que evalúa el cumplimiento de los porcentajes de emisión accesible establecidos por la citada Ley General de la Comunicación Audiovisual; para el ejercicio de 2017, prácticamente todos los canales contemplados cumplieron con los porcentajes referidos a la emisión de SPS (un mínimo de un $90 \%$ para los canales públicos y al menos el $75 \%$ de su programación para los operadores privados) (CNMC 2019).

Asimismo, la norma UNE 153010 (2012) de Subtitulado para personas sordas y personas con discapacidad auditiva, elaborada por la Asociación Española de Normalización y Certificación (AENOR 2012), recoge los parámetros básicos que debe respetar una SPS que se considere de calidad y, de hecho, será esta norma la que se estudie en profundidad en la asignatura de accesibilidad en la que se ha llevado a cabo el presente proyecto. No obstante, si bien esta norma supone un excelente referente para la práctica de la SPS, aún resulta ambigua en muchos aspectos y no atiende a la enorme diversidad de perfiles de personas con algún tipo de deficiencia auditiva que se incluyen en la población española (Báez Montero y Fernández Soneira 2010). Por lo tanto, en numerosas ocasiones esta norma puede resultar insuficiente, tal como ya sucedía con su predecesora, la norma UNE 153010 (2003) (Pereira Rodríguez y Lorenzo García 2005). Frente a este panorama, además de la competencia traductora general (Kelly 2005) y lingüística (Díaz Cintas 2006) en particular que deben desarrollar los estudiantes, se considera imprescindible inculcar un espíritu crítico y proactivo en los futuros profesionales de la SPS, para que sean capaces de atender a las necesidades del público con sordera y tomar decisiones beneficiosas para los consumidores.

Por último, cabe hacer un breve repaso a las guías docentes disponibles en la web de los grados en Traducción e Interpretación que se imparten en las universidades públicas españolas en la actualidad para comprobar que, al menos sobre el papel, la concienciación de los estudiantes sobre las particularidades y necesidades de la comunidad sorda no ocupa un espacio especialmente relevante en los planes de estudio. En concreto, en la siguiente tabla recogemos información sobre los sitios web de las 18 universidades públicas en las que se imparte esta titulación, según el Libro blanco del grado en Traducción e Interpretación (ANECA 2004). Excluimos las universidades privadas y los títulos de posgrado. La información de esta tabla procede exclusivamente de los contenidos disponibles en la web. 
Tabla 1. Universidades públicas españolas que ofertan el grado en Traducción e Interpretación e inclusión de contenidos sobre accesibilidad en las guías docentes

\begin{tabular}{|c|c|c|c|}
\hline Universidad & $\begin{array}{l}\text { Asignatura relacionada con } \\
\text { la accesibilidad/TAV }\end{array}$ & $\begin{array}{l}\text { Contenidos } \\
\text { específicos de } \\
\text { accesibilidad }\end{array}$ & $\begin{array}{l}\text { Referencias a los } \\
\text { perfiles de la } \\
\text { comunidad sorda }\end{array}$ \\
\hline $\begin{array}{l}\text { Universidad de } \\
\text { Granada }\end{array}$ & $\begin{array}{l}\text { Traducción multimedia inglés } \\
\text { (obligatoria, } 4 .^{\circ} \text { ) }\end{array}$ & No & No \\
\hline $\begin{array}{l}\text { Universidad de } \\
\text { Córdoba }\end{array}$ & $\begin{array}{l}\text { - Traducción cinematográfica } \\
\text { y subtitulado (obligatoria, } 4^{\circ} \text { ) } \\
\text { - Traducción multimedia } \\
\left(\text { optativa, } 4 .^{\circ} \text { ) }\right.\end{array}$ & No & No \\
\hline $\begin{array}{l}\text { Universidad de } \\
\text { Málaga }\end{array}$ & $\begin{array}{l}\text { Traducción Audiovisual } \\
\left.\text { BA-AB (obligatoria, } 3 .^{\circ}\right)\end{array}$ & No & No \\
\hline $\begin{array}{l}\text { Universidad } \\
\text { Pablo de Olavide }\end{array}$ & $\begin{array}{l}\text { Traducción audiovisual } \\
\left(\text { optativa, } 4 . .^{\circ}\right)\end{array}$ & $\begin{array}{l}\text { Sí: Tema } 3 . \text { La } \\
\text { accesibilidad } \\
\text { en los medios }\end{array}$ & $\begin{array}{l}\text { Sí: Recepción y } \\
\text { audiencia en TAV }\end{array}$ \\
\hline $\begin{array}{l}\text { Universidad de } \\
\text { Salamanca }\end{array}$ & $\begin{array}{l}\text { Traducción Audiovisual } \\
\left(\text { optativa, } 4 .^{\circ} \text { ) }\right.\end{array}$ & No & No \\
\hline $\begin{array}{l}\text { Universidad de } \\
\text { Valladolid }\end{array}$ & $\begin{array}{l}\text { Lenguajes visuales (optativa, } \\
\left.4^{\circ} .^{\circ}\right)\end{array}$ & No & No \\
\hline $\begin{array}{l}\text { Universitat } \\
\text { Autònoma de } \\
\text { Barcelona }\end{array}$ & $\begin{array}{l}\text { Traducción audiovisual y } \\
\text { localización (optativa, } 4 .^{\circ} \text { ) }\end{array}$ & No & No \\
\hline $\begin{array}{l}\text { Universitat } \\
\text { Pompeu Fabra }\end{array}$ & $\begin{array}{l}\text { - Traducción audiovisual } \\
\text { (optativa, } 3 .^{\circ} \text { ) } \\
\text { - Taller de Traducción y } \\
\text { Medios de Comunicación } \\
\text { (optativa, } 3 .^{\circ} \text { ) }\end{array}$ & No & $\begin{array}{l}\text { No } \\
\text { Sí: Conocer las } \\
\text { necesidades } \\
\text { específicas de las } \\
\text { personas con } \\
\text { habilidades } \\
\text { sensoriales especiales }\end{array}$ \\
\hline $\begin{array}{l}\text { Universidad } \\
\text { Autónoma de } \\
\text { Madrid }\end{array}$ & No & No & No \\
\hline $\begin{array}{l}\text { Universidad } \\
\text { Complutense de } \\
\text { Madrid }\end{array}$ & $\begin{array}{l}\text { - Mediación Lingüística para } \\
\text { la Accesibilidad (optativa, } 3 .^{\circ} \text { ) } \\
\text { - Técnicas para la TAV B1/ } \\
\text { B2-A (optativa, } 3 .^{\circ} \text { ) } \\
\text { - Traducción Audiovisual } \\
\text { B1-A (optativa, } \text { 4. }^{\circ} \text { ) }\end{array}$ & $\begin{array}{l}\text { Sí } \\
\text { No } \\
\text { No }\end{array}$ & $\begin{array}{l}\text { Sí (?): Mediación } \\
\text { lingüística para } \\
\text { personas con } \\
\text { disfunción sensorial. } \\
\text { No } \\
\text { No }\end{array}$ \\
\hline $\begin{array}{l}\text { Universidad Rey } \\
\text { Juan Carlos }\end{array}$ & No & No & No \\
\hline $\begin{array}{l}\text { Universitat } \\
\text { d'Alacant }\end{array}$ & TAV B-A/A-B (optativa, $3 .^{\circ}$ ) & No & No \\
\hline $\begin{array}{l}\text { Universitat de } \\
\text { València }\end{array}$ & $\begin{array}{l}\text { - Traducción especializada } \\
\text { (oblig., } 4 .^{\circ} \text { ) }\end{array}$ & Sí & No \\
\hline $\begin{array}{l}\text { Comun. } \\
\text { Valenciana }\end{array}$ & $\begin{array}{l}\text { - Las profesiones del traductor } \\
\text { (optativa, } 4 .^{\circ} \text { ) }\end{array}$ & No & No \\
\hline
\end{tabular}




\begin{tabular}{|c|c|c|c|}
\hline Universidad & $\begin{array}{l}\text { Asignatura relacionada con } \\
\text { la accesibilidad/TAV }\end{array}$ & $\begin{array}{l}\text { Contenidos } \\
\text { específicos de } \\
\text { accesibilidad }\end{array}$ & $\begin{array}{l}\text { Referencias a los } \\
\text { perfiles de la } \\
\text { comunidad sorda }\end{array}$ \\
\hline $\begin{array}{l}\text { Universitat } \\
\text { Jaume I }\end{array}$ & $\begin{array}{l}\text { Traducción Audiovisual y } \\
\text { Accesibilidad (optativa, } \text { 4. }^{\circ} \text { ) }\end{array}$ & $\begin{array}{l}\text { Sí: SPS - El } \\
\text { receptor sordo } \\
\text { o con } \\
\text { discapacidades } \\
\text { auditivas }\end{array}$ & $\begin{array}{l}\text { Sí: Ser capaz de } \\
\text { identificar y cubrir las } \\
\text { necesidades del } \\
\text { receptor con } \\
\text { problemas auditivos o } \\
\text { de visión. }\end{array}$ \\
\hline $\begin{array}{l}\text { Universidade de } \\
\text { Vigo }\end{array}$ & $\begin{array}{l}\text { Traducción medios } \\
\text { audiovisuales (optativa, } 4^{\circ} \text { ) }\end{array}$ & No & No \\
\hline $\begin{array}{l}\text { Universidad de } \\
\text { las Palma de } \\
\text { Gran Canaria }\end{array}$ & $\begin{array}{l}\text { Traducción audiovisual y } \\
\text { accesibilidad (obligatoria, 3. } .^{\circ} \text { ) }\end{array}$ & Sí & $\begin{array}{l}\text { Sí: Unidad } 6 . \\
\text { Introducción a la SPS: } \\
\text { las barreras de } \\
\text { comunicación para las } \\
\text { personas con } \\
\text { discapacidad auditiva. }\end{array}$ \\
\hline $\begin{array}{l}\text { Universidad de } \\
\text { Murcia }\end{array}$ & $\begin{array}{l}\text { Traducción especializada } \\
\text { audiovisual (obligatoria, } 4 .^{\circ} \text { ) }\end{array}$ & Sí & No consta \\
\hline $\begin{array}{l}\text { Universidad del } \\
\text { País Vasco }\end{array}$ & No & No & No \\
\hline
\end{tabular}

Como puede comprobarse, de las 18 universidades cuyas guías docentes han podido consultarse en la web para los cursos académicos 2018/2019 y 2019/2020, 15 de ellas ofertan asignaturas de o relacionadas con la accesibilidad; 7 ofrecen formación específica en accesibilidad. Sin embargo, en los contenidos y objetivos de únicamente 5 asignaturas parece hacerse referencia a los perfiles de las personas sordas y, por tanto, a la concienciación del alumnado de las particularidades de la audiencia para la que trabajarán.

\section{Metodología del proyecto de innovación docente}

\subsection{Objetivos}

Dado este estado de la cuestión y ante las competencias básicas en las que queremos hacer hincapié para enriquecer la formación de subtituladores (Tamayo Masero 2016: 329),

y dado el auge que están experimentando la práctica, la docencia y la investigación en la disciplina de accesibilidad y la SPS, parece ineludible establecer un diálogo continuo entre estos tres ámbitos, que dé cuenta de las necesidades reales del colectivo con discapacidad auditiva y de su continuo cambio.

Así, se ha considerado útil plantear un proyecto que cuente con la participación en el aula de SPS de dos personas con deficiencias auditivas, cuyos principales objetivos son: 
1. Desarrollo de la competencia interpersonal en los profesionales de SPS.

2. Concienciación sobre las distintas necesidades de los receptores sordos.

3. Desarrollo de la capacidad crítica de las prácticas actuales en SPS.

Para cumplir estos objetivos principales, se planificó una sesión en el aula centrada en tres tipos de actividades, directamente relacionados con los tres objetivos anteriores:

1. Contacto directo con receptores reales de los productos audiovisuales subtitulados (dos mujeres) y elaboración de tareas en equipo por parte del alumnado.

2. Presentación y comparación de los perfiles de las personas sordas y análisis de sus observaciones ante la visualización de varios clips de vídeo con SPS.

3. Debate y valoración de las directrices de la norma UNE en función de las observaciones hechas en clase.

Ante el pequeño alcance temporal y económico del que se disponía, este proyecto de innovación se desarrolló durante una única sesión, aunque se extendió con actividades programadas para preparar la sesión y posteriores a ella que completaran los objetivos principales (apartado 3.2.). Asimismo, aunque sería muy beneficioso poder contar con más personas del colectivo sordo, el alcance económico del proyecto se ajustó a la presencia de dos personas. Al tratarse de una experiencia piloto, se consideran suficientes para valorar el éxito de un primer contacto entre el colectivo sordo y el alumnado, que podrá ampliarse en el futuro. Cabe señalar además que, si bien es cierto que una sola clase no nos permite llevar a cabo un proyecto de innovación de gran envergadura, los resultados positivos que se observaron y que se expondrán a continuación evidencian un excelente punto de partida para ampliar este proyecto con más actividades de interlocución con los futuros receptores de los productos audiovisuales subtitulados, de manera que los alumnos reciban una formación integral y concienciada de las necesidades que habrán de cubrir con su práctica profesional.

Este proyecto se basa parcialmente en la metodología de aprendizaje-servicio (ApS), concebida «como un método pedagógico basado en la experiencia, en el que el proceso de enseñanza-aprendizaje radica en la aplicación de lo que se va aprendiendo ante necesidades sociales reales» (Chiva-Bartoll, Pallarés-Piquer y Gil-Gómez 2018: 183). Según esto,

los estudiantes trabajan contenidos y objetivos curriculares [...]. El ApS implica una participación activa del alumnado en los diferentes momentos del proceso. [...] El necesario proceso de reflexión que implica la participación en programas de ApS permite a los estudiantes conocerse no solo a sí mismos, sino también conocer e interactuar con sus compañeros, potenciando enormemente la empatía, las habilidades de comunicación y, en definitiva, las estrategias para optimizar su interacción con el profesorado, con los receptores del servicio y con los diferentes agentes implicados. 
Si bien con este proyecto empleamos la metodología ApS en la fase de concienciación y reflexión, la labor de prestar un servicio significativo a la comunidad se reserva para una ampliación futura del proyecto, mediante la cual sean los propios alumnos quienes elaboren un producto audiovisual accesible basado en su experiencia de interacción con los receptores sordos en el aula.

Por otra parte, también se recurrió a una perspectiva cualitativa en la que los alumnos expusieron su valoración final de la sesión y las implicaciones que las actividades tuvieron para su formación como futuros profesionales de la SPS. Asimismo, se llevaron a cabo entrevistas semiestructuradas con las personas invitadas al aula. Tales entrevistas se construyeron mediante la colaboración de todos los estudiantes y la extracción de las cuestiones comunes que todos los grupos plantearon, de forma que fueron ellos los protagonistas tanto del desarrollo de la sesión como de la preparación y análisis de buena parte de los materiales que se emplearon durante la clase. El papel de la profesora se limitó a la moderación y al agrupamiento de preguntas cuando se detectaran elementos repetitivos o ya formulados. Se trata por tanto de ajustarnos al modelo de docencia basado en convertir al alumno en el centro de su propio aprendizaje (González Davies 2004).

\subsection{Actividades}

\subsubsection{Actividad previa}

La asignatura de accesibilidad en el curso 2018-2019 contó con 50 alumnos matriculados. El proyecto se dividió en tres etapas fundamentales: una actividad previa de preparación a la sesión en clase, dividida en tres tareas, que realizaron los alumnos en grupos de 3-4 personas; la clase con la participación de dos asociadas de ASPAS (Asociación de Familias y Personas Sordas de la Provincia de Castellón), que acudieron voluntariamente, acompañadas de la Coordinadora de Proyectos y Servicios de ASPAS-Castellón; y, finalmente, una actividad de reflexión posterior que también elaboraron los alumnos en grupo, a partir de la experiencia de clase.

Figura 1. Fases del proyecto
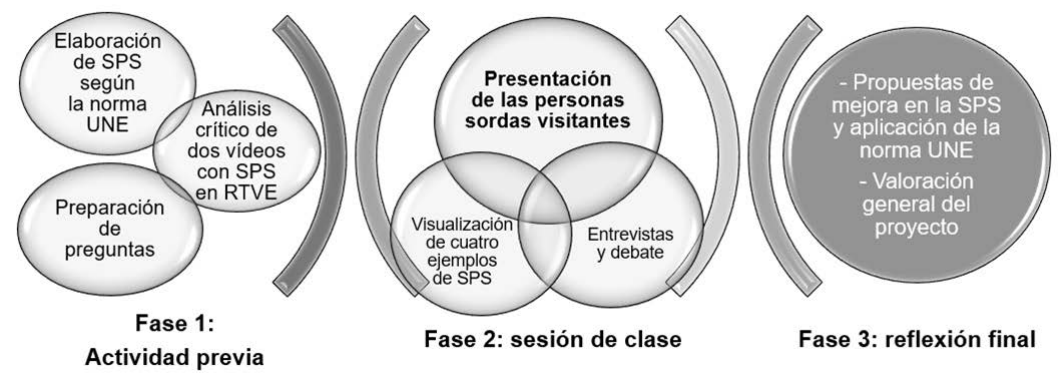

Para la tarea inicial, los alumnos recibieron las instrucciones de visualizar dos cortos cómicos disponibles a través de la web de Radiotelevisión Española (RTVE) mediante el servicio «a la carta» (<http://www.rtve.es/alacarta/>). Este 
servicio ofrece buena parte de su programación con subtítulos, aunque no se especifica de qué tipo de subtítulos se trata, simplemente se califican de «subtítulos en castellano». En concreto, los vídeos, de aproximadamente 4-5 minutos, pertenecían a la webserie Neverfilms (PLAYZ.ES 2017), producida para Playz, la plataforma de contenidos digitales de RTVE. Se escogieron estos dos vídeos por tratarse de parodias breves de tráileres de películas, cuyo contenido cómico era evidente y podría comprobarse fácilmente si los usuarios sordos que iban a ver los vídeos durante la sesión de clase disfrutaban de la parodia sin dificultad. Una vez los alumnos vieron estos dos tráileres, se les pidió que elaboraran una reflexión sobre qué aspectos contenidos en estos subtítulos no se correspondían con las indicaciones de la norma UNE 153010 (2012), presentada y trabajada ya en profundidad en dos sesiones de clase previamente.

Con respecto a esta primera actividad de visualización y análisis de los dos vídeos de RTVE, basada en el trabajo colaborativo (grupos de unas 3-4 personas), el objetivo que se persiguió fue fomentar el espíritu crítico y la capacidad de evaluación, por parte de los alumnos, de un producto que debía cumplir con las directrices que se irían trabajando a lo largo de la asignatura. La evaluación crítica es una herramienta muy útil para el alumnado, que, de esta forma, desempeña un papel activo en su propio proceso de aprendizaje y puede ser más consciente de aspectos positivos y negativos (De Higes Andino y Cerezo Merchán 2018) del producto que evalúa y que habrá de aprender a elaborar durante la asignatura.

Una segunda tarea incluida en esta primera actividad en grupo fue la redacción de una serie de preguntas que consideraran adecuadas para comprobar la satisfacción de los receptores sordos frente a los subtítulos emitidos por RTVE y la comprensión de los dos vídeos anteriores. Con ello, se situó al alumnado como protagonista del desarrollo de contenidos que se utilizarían durante la sesión de clase con las personas sordas, puesto que fueron ellos los encargados de elaborar el listado guía de preguntas de las entrevistas semiestructuradas. Del listado que proporcionaron los 14 grupos de alumnos se extrajeron 27 preguntas, distribuidas en 8 bloques, cada uno relativo a un aspecto de la SPS contemplado en la norma UNE: aspectos visuales, temporales, identificación de personajes, efectos sonoros, información contextual, música y canciones, criterios editoriales y otras cuestiones.

Asimismo, como última tarea preparatoria para la sesión y también para profundizar en el conocimiento de la norma UNE 153010, se les pidió a los alumnos, en los mismos grupos de trabajo, que elaboraran la SPS de un fragmento de una película aplicando todas las directrices de dicha norma. Esta subtitulación la entregaron a través del aula virtual de la asignatura en formato ASS.

El papel de la profesora en esta fase fue el de recopilar y agrupar todas las preguntas propuestas por los 14 grupos de alumnos, así como visualizar y escoger una de las subtitulaciones entregadas por ellos para proyectarla posteriormente en clase. Se eligió una SPS al azar que contenía algunos errores, pero también varios aciertos.

\subsubsection{Actividad central}

Tras la actividad preparatoria fuera del aula, la sesión de dos horas de clase fue el elemento central del proyecto: contamos con la participación de dos mujeres con 
grados distintos de deficiencias auditivas y una coordinadora de servicios de la asociación ASPAS de Castellón. Para comenzar la sesión, esta coordinadora ofreció una introducción a los alumnos sobre las labores que desempeñan en la asociación, así como los distintos grados de sordera que pueden presentar sus miembros, la diversidad de perfiles que los caracteriza y los distintos métodos que emplean para comunicarse; en concreto, los audífonos y el sistema de frecuencia modulada (FM) (Tamayo Masero 2015). A continuación, las dos personas con deficiencias auditivas se presentaron, explicaron el tipo de sordera que experimentan, sus preferencias en el consumo de productos audiovisuales y todos los detalles que consideraron de interés sobre su perfil personal como parte del colectivo sordo y sobre su vida diaria.

Tras ello, se realizaron dos tareas en clase: en primer lugar, la visualización de los primeros cinco minutos de una película subtitulada para sordos perteneciente al catálogo de servicios de ASPAS. Esta película la aportó la coordinadora de ASPAS, aunque no pudo confirmar de quién era la autoría de la SPS que contenía. Tras ello, se vieron también los dos tráileres cómicos cuya subtitulación, elaborada para RTVE, habían analizado los alumnos previamente. Tras ambas visualizaciones, se recurrió al listado de preguntas extraídas de las propuestas de los alumnos para entrevistar a las personas sordas sobre el nivel de satisfacción y comprensión que mostraron tras cada uno de los vídeos (el fragmento de la película de ASPAS y los dos tráileres de RTVE). El listado de preguntas de los alumnos, por tanto, se empleó a modo de entrevista semiestructurada (Kvale y Brinkmann 1996) para guiar a los alumnos en su interlocución con las visitantes. Asimismo, los alumnos comentaron con las entrevistadas los análisis que ellos mismos elaboraron evaluando la aplicación o no de la norma UNE en el material analizado, de forma que se estableció un debate entre la idoneidad de los aspectos contemplados en dicha norma y la satisfacción de las usuarias.

Por último, se proyectó también uno de los archivos de subtítulos elaborados por los alumnos como tarea previa. Tras ello, de nuevo, se comentaron los aspectos positivos y mejorables. De este modo, volvió a establecerse una interacción continua con las visitantes, que expresaron su grado de satisfacción con la SPS que vieron, así como una evaluación en conjunto por parte de los alumnos de un trabajo elaborado por ellos mismos.

\subsubsection{Actividad posterior}

Una vez concluida la sesión de clase, la tercera etapa del proyecto, ya fuera del aula, se basó en la elaboración de una reflexión, también en los mismos grupos iniciales de 3-4 personas, sobre el perfil que presentaba cada una de las dos personas sordas que acudieron a clase y qué cambios consideraban los alumnos que se podrían realizar en la norma UNE para atender las necesidades del público con deficiencias auditivas. También se les pidió una breve valoración de la sesión de clase y la utilidad de haber conocido a estas dos consumidoras de productos audiovisuales subtitulados. 


\section{Resultados}

El trabajo de los alumnos durante la fase previa a la visita de las personas sordas evidenció un espíritu crítico y un compromiso significativo con la calidad de la SPS: en la valoración cualitativa del uso de la norma UNE en los subtítulos que analizaron, el alumnado detectó numerosos aspectos de los distintos bloques contemplados en dicha norma que no se cumplían en los subtítulos emitidos en la web de RTVE. En especial, indicaron la falta de sincronía en algunos casos, dada la cantidad de diálogo y la velocidad a la que este se escucha, y la literalidad de los subtítulos, que los hacía en ocasiones demasiado extensos, así como la colocación de los efectos sonoros, que en ningún caso aparecen en posición 9 como sí recomienda la citada norma. También se llamó la atención sobre la falta de homogeneidad, tanto en el uso de guiones como en la asignación de colores, para identificar a los personajes. Asimismo, algunos grupos incluyeron en sus análisis propuestas puntuales de mejora en los subtítulos analizados para ajustarse más a las directrices de la norma UNE, como la inserción de etiquetas con algunas intervenciones en off o la síntesis de ciertas oraciones para ajustarse a los parámetros recomendados de espacio-tiempo.

Por otra parte, prácticamente todos los grupos coincidieron en plantear preguntas para los receptores sordos con respecto a la adecuada temporización de los subtítulos, al empleo de colores para la identificación de personajes y a la facilidad de lectura y comprensión del vídeo, y en muchos casos también incluyeron preguntas sobre la posición de los subtítulos y los efectos sonoros. Por ello, se consideró que lo más apropiado para reunir todas estas preguntas en un guion estructurado y lógico era seguir la distribución de contenidos de la norma UNE en torno a ocho bloques: aspectos visuales, aspectos temporales, identificación de personajes, efectos sonoros, información contextual, música y canciones, criterios editoriales y otras cuestiones. En total, se obtuvieron 27 preguntas que sirvieron para estructurar el diálogo entre el alumnado y las dos personas visitantes.

Durante la sesión con las personas sordas en clase, los alumnos se mostraron muy comprometidos, con un elevado nivel de participación, ya que fueron protagonistas en la elaboración de las entrevistas semiestructuradas y también habían analizado previamente buena parte del material que se visualizó en clase y estaban familiarizados con las directrices de la norma UNE, lo cual los ayudó a discutir los aciertos y mejoras del material con mayor soltura. También parecieron sentirse cómodos pudiendo recurrir a un listado previo de preguntas que orientara su interacción con las dos personas sordas.

Aunque esta única sesión no contó con una evaluación específica, sí se incluyó, como se ha explicado, una valoración posterior para determinar la utilidad de las tareas realizadas. En las reflexiones y comentarios posteriores al proyecto, los alumnos demostraron haber asimilado la diversidad de perfiles que podrán encontrarse de personas con deficiencias auditivas, ya que trazaron con detalle el perfil de cada una de las dos visitantes y mostraron su inquietud con la falta de atención a la diversidad que parece desprenderse de la norma UNE, por motivos prácticos. Prácticamente todos los grupos indicaron que les resultó muy útil conocer de pri- 
mera mano la opinión de dos consumidoras de productos audiovisuales con SPS, ya que pudieron plantearles preguntas que resultan ambiguas según las directrices de la norma UNE. Además, dado que con tan solo dos personas las respuestas que recibieron de cada una de ellas fueron diferentes, todos señalaron que les llamó la atención descubrir la diversidad de perfiles y necesidades que pueden presentar las personas con deficiencias auditivas. Un trato directo con ellas pareció concienciarlos más aún que la mera referencia a la heterogeneidad de la comunidad sorda, sin ejemplos reales. Las siguientes opiniones provienen de los comentarios que incluyeron dos de los 14 grupos de trabajo en su reflexión:

Grupo 1:

En último lugar, me gustaría explicar por qué me ha resultado útil el contacto con ellas. Cuando realizas un trabajo que está dirigido a un tipo de personas concreto, siempre resulta beneficioso estar en contacto con esas personas y saber con exactitud a qué perfil te estás enfrentando. Esto no solo sirve para dar sentido a tu trabajo, sino también para mejorar como profesional, ya que podrás identificar con mayor facilidad cuáles son sus necesidades. También me gustaría comentar que me ha parecido muy útil que vieran con nosotros dos vídeos subtitulados por esa misma razón: puedes ver exactamente cuál es su reacción y si el acto comunicativo se ha completado con éxito.

Grupo 2:

Creemos que esta sesión ha sido muy interesante. En primer lugar, porque hemos podido conocer de primera mano la experiencia de personas con deficiencias auditivas, lo que nos ha ayudado a comprender sus necesidades y preferencias. Sin embargo, también nos ha servido para darnos cuenta de que la norma UNE, aunque hecha con buena intención, no siempre recoge lo que a todo el público no oyente le gustaría. Esto ocurre porque cada persona es un mundo y sus preferencias dependen en gran medida de cuánto tiempo llevan teniendo problemas de audición y de la frecuencia con la que consumen productos audiovisuales accesibles.

Por otra parte, también resultó interesante comprobar que las dos personas sordas entrevistadas no conocían la norma UNE ni sus directrices, aunque sí son consumidoras habituales de productos audiovisuales accesibles. En general, expresaron su satisfacción con los subtítulos que vieron en clase, aunque preferían la inclusión de algo más de información en el producto audiovisual, por tanto, que los subtítulos fueran lo más fieles posible a los diálogos orales, y que se explicitara también todo efecto sonoro (entiéndase esta como la preferencia de únicamente dos personas que consumen productos audiovisuales). Esto, como ya se apuntaba en estudios previos (González Davies 2004), parece estar en contradicción con una correcta comprensión del producto. Dado que la velocidad de lectura se elevaría considerablemente por encima de los 14-18 caracteres por segundo recomendables, las opiniones de los usuarios no siempre deben tomarse como única referencia a la hora de aplicar determinadas decisiones de traducción. En cualquier caso, el mensaje principal que extrajeron los alumnos, como se pretendía, fue la importancia de conocer al público para el que trabajarán y, en el 
caso de la comunidad sorda, el amplio abanico de perfiles con los que pueden encontrarse y la flexibilidad que necesitarán como subtituladores para adaptar su trabajo a las expectativas de los usuarios.

\section{Conclusiones}

Tras completar el presente proyecto de innovación docente, concluimos que se han cumplido los tres objetivos iniciales a partir de los siguientes indicadores:

1. Desarrollo de la competencia interpersonal en los profesionales de SPS: los alumnos completaron tareas previas y posteriores al proyecto con un elevado nivel de calidad e incluyendo reflexiones críticas beneficiosas para su formación. Durante la sesión de clase, se establecieron diálogos y debates sobre la idoneidad de los subtítulos analizados y sobre posibilidades de mejora. Las personas invitadas con deficiencias auditivas expresaron libremente sus preferencias y los alumnos recogieron estas opiniones para la tarea final y para futuros proyectos.

2. Concienciación sobre las distintas necesidades de los receptores sordos: los alumnos supieron establecer una interacción fluida y adecuada con los invitados sordos al aula, mostraron interés y absoluto respeto por las preferencias que estos expresaron y recogieron propuestas de mejora en las reflexiones finales.

3. Desarrollo de la capacidad crítica de las prácticas actuales en subtitulación para personas sordas: los alumnos fueron capaces de valorar de forma crítica la adecuación de material audiovisual con subtítulos emitido en una cadena pública, así como de subtítulos creados por ellos mismos y por la asociación invitada ASPAS. Del mismo modo, plasmaron sus impresiones y propuestas de mejora en las tareas finales que elaboraron en grupo.

Como se ha indicado al comienzo, este proyecto representa un reducido, aunque prometedor punto de partida para ampliar la metodología de aprendizaje-servicio en el futuro, incluyéndola en la docencia de la accesibilidad mediante proyectos de subtitulación para personas con deficiencias auditivas que puedan emitirse y analizar su recepción en una audiencia mucho más amplia. Esta actividad de innovación educativa ha resultado exitosa para trabajar la competencia interpersonal en el aula de SPS, así como para concienciar a los alumnos de la necesidad de conocer a su público potencial y las peculiaridades que lo caracterizan. Se trata así de contribuir a ofrecerles una formación integral que contemple todas las competencias propias de la formación de los profesionales de la TAV, con la particularidad, en el caso de la SPS, de que estos profesionales elaborarán un producto para una audiencia con unas características sensoriales y sociales muy determinadas que será necesario conocer en profundidad (Neves 2008). 


\section{Referencias bibliográficas}

AENOR (2012). Norma español UNE 153010: Subtitulado para personas sordas y con discapacidad auditiva. Madrid.

ANECA (2004). Libro blanco del título de grado en Traducción e Interpretación. Agencia Nacional de la Evaluación de la Calidad y Acreditación.

Arumí Ribas, M.; Romero-Fresco, P. (2008). «A Practical Proposal for the Training of Respeakers». JosTrans. The Journal of Specialised Translation, 10, p. 106-127.

Badia Cardús, A.; Matamala, A. (2007). «La docencia en accesibilidad en los medios». TRANS. Revista de Traductología, 11, p. 61-72.

Báez Montero, I. C.; Fernández Soneira, A. M. (2010). «Spanish deaf people as recipients of closed captioning». En: Matamala, A.; Orero, P. (eds.). Listening to subtitles: Subtitles for the deaf and hard of hearing. Nueva York: Peter Lang.

Cerezo Merchán, B.; De Higes Andino, I. (2013). «Trabajo colaborativo y desempeño profesional: un caso práctico en la clase de accesibilidad audiovisual». Hikma, 12, p. 65-85.

Chiva-Bartoll, Ó.; Pallarés-Piquer, M.; Gil-Gómez, J. (2018). «Aprendizaje-servicio y mejora de la personalidad eficaz en futuros docentes de educación física». Revista Complutense de Educación, 29 (1), p. 181-197.

CNMC (Comisión Nacional de los Mercados y la Competencia) (2019). Informe sobre el seguimiento de las obligaciones impuestas en materia de accesibilidad correspondiente al año 2017. Madrid.

Consejo de Gobierno, claustro (2016). Modelo educativo de la UJI. Castellón de la Plana.

Díaz Cintas, J. (2006). Competencias profesionales del subtitulador y el audiodescriptor. Getafe. (en línea) <https://www.cesya.es/sites/default/files/documentos/informe_ formacion.pdf>.

- (2010). «La accesibilidad a los medios de comunicación audiovisual a través del subtitulado y de la audiodescripción». En: González, L.; Hernúñez, P. (eds.). El español, lengua de traducción para la cooperación y el diálogo. Instituto Cervantes, p. 157-180.

Díaz-Cintas, J.; Orero, P; Remael, A. (2007). Media for all: Subtitling for the deaf, audio description, and sign language. Nueva York: Rodopi.

González Davies, M. (2004). Multiple voices in the translation classroom: Activities, tasks and projects. Filadelfia: John Benjamins.

De Higes Andino, I.; Cerezo Merchán, B. (2018). «Using evaluation criteria and rubrics as learning tools in subtitling for the D/deaf and the hard of hearing». The Interpreter and Translator Trainer, 12 (1), p. 68-888.

Ivarsson, J.; Carroll, M. (1998). Subtitling. Simrishamn: TransEdit HB.

Kelly, D. (2005). A Handbook for translator trainers: A guide to reflective practice. Northampton, Massachusetts: St. Jerome.

Kvale, S.; Brinkmann, S. (1996). Interviews: An introduction to qualitative research interviewing. 2nd ed. Los Angeles: Sage Publications.

Neves, J. (2005). Audiovisual translation: Subtitling for the deaf and hard of hearing. Tesis doctoral. Roehampton University.

- (2008). «Training in Subtitling for the d/Deaf and the Hard of Hearing». En: Díaz CinTAs, J. (ed.). The Didactics of Audiovisual Translation. Ámsterdam: John Benjamins, p. 171-189.

Pereira Rodríguez, A. M. (2005). «El subtitulado para sordos: Estado de la cuestión en España». Quaderns. Revista de Traducció, 12, p. 161-172. 
Pereira Rodríguez, A. M.; Lorenzo García, L. (2005). «Evaluamos la norma UNE 153010: Subtitulado para personas sordas y personas con discapacidad auditiva. Subtitulado a través del teletexto». Puentes, 4, p. 21-26.

PLAYZ.ES (2017). «Arranca el rodaje de 'Neverfilms', el nuevo formato de humor de Playz, Corporación de Radio y Televisión Española». (en línea) <http://www.rtve.es/ playz/20171124/arranca-rodaje-neverfilms-nuevo-formato-humor-playz/1638283. shtml> (Consulta: 22 marzo 2020).

ReVERTER Oliver, B. (2019). Inclusión del alumnado con discapacidad sensorial y traducción audiovisual en las aulas de inglés de las EEOOII de la Comunitat Valenciana: Un estudio exploratorio. Universitat de València.

RodríGuez Esteban, A. (2007). «Las competencias en el Espacio Europeo de Educación Superior. Tipologías». Humanismo y Trabajo Social, 6 (139), p. 139-153.

Tamayo Masero, A. (2015). Estudio descriptivo y experimental de la subtitulación en TV para niños sordos. Una propuesta alternativa. Universitat Jaume I.

- (2016). «Subtitulación para personas sordas: metodología de aprendizaje-servicio en el aula de traducción audiovisual». Ikala, 21 (3), p. 327-342. 